\title{
Patient-centred care in thoracic surgery: a holistic approach - A review of the subjects of enhanced recovery after surgery, rehabilitation, pain management and patient-reported outcome measures in thoracic surgery
}

\author{
Alessandro Brunelli ${ }^{1 \wedge}$, Shanda H. Blackmon ${ }^{2} \wedge$, Mert Sentürk ${ }^{3}$, Vinicius Cavalheri ${ }^{4,5}$, Cecilia Pompili $^{6,7} \wedge$ \\ ${ }^{1}$ Department of Thoracic Surgery, St. James's University Hospital, Leeds, UK; ${ }^{2}$ Division of Thoracic Surgery, Mayo Clinic Rochester, Rochester, \\ Minnesota, USA; ${ }^{3}$ Department of Anesthesiology and Intensive Care, Istanbul Medical Faculty, Istanbul, Turkey; ${ }^{4}$ Curtin School of Allied Health, \\ Curtin University, Perth, Western Australia, Australia; ${ }^{5}$ Allied Health, South Metropolitan Health Service, Perth, Western Australia, Australia; \\ ${ }^{6}$ Patient Centred Outcomes Research (PCOR), University of Leeds, Leeds, UK; ${ }^{7}$ Thoracic Surgery Unit, University Hospital Trust-Ospedale Borgo \\ Trento, Verona, Italy \\ Contributions: (I) Conception and design: All authors; (II) Administrative support: None; (III) Provision of study materials or patients: None; (IV) \\ Collection and assembly of data: All authors; (V) Data analysis and interpretation: None; (VI) Manuscript writing: All authors; (VII) Final approval of \\ manuscript: All authors. \\ Correspondence to: Dr. Alessandro Brunelli. Department of Thoracic Surgery, St. James's University Hospital Bexley Wing, Beckett Street, Leeds, LS9 \\ 7TF, UK. Email: alexit_2000@yahoo.com.
}

\begin{abstract}
The symposium Patient-centered care in thoracic care: a bolistic approach was held on June 22, 2021, as a virtual event in the context of the European conference on general thoracic surgery. Its aim was to explore how to improve patient outcomes in thoracic surgery by using enhanced recovery after surgery (ERAS®) frameworks and collaboration within healthcare. During the four presentations, topics on patient mobilization and physical rehabilitation, pain management, and the role of chest drainage in facilitating perioperative care were discussed. Strategies to minimize opioid consumption and incorporate patients' experiences as quality indicators were described. There were two main ideas that were considered pivotal to achieve optimal care: (I) the use of simple, easily implementable perioperative protocols and practices to improve compliance from both patients and the healthcare team, and (II) promote a better recovery with early mobilization and reducing the patient's levels of pain. Among the key learnings that emerged from the presentations are the importance of taking the patient's experiences into account, including what they value the most after surgery, and how technology can enable better care. The use of digital chest drainage systems emerged as a way for improving patient outcomes and experiences across several key indicators.
\end{abstract}

Keywords: Enhanced recovery after surgery (ERAS®); patient mobilization; pain management; patient-reported outcomes and experiences; digital chest drainage systems

Submitted Nov 16, 2021. Accepted for publication Jan 07, 2022.

doi: $10.21037 /$ jtd-21-1763

View this article at: https://dx.doi.org/10.21037/jtd-21-1763

^ ORCID: Alessandro Brunelli, 0000-0002-6505-1656; Shanda H. Blackmon, 0000-0003-3639-9941; Vinicius Cavalheri, 0000-0001-8620-

7499; Cecilia Pompili, 0000-0001-6746-5441. 


\section{Background}

The virtual symposium Patient-centered care in thoracic care: a holistic approach was held on June 22, 2021, as a part of the $29^{\text {th }}$ meeting of the European Society of Thoracic Surgeons (ESTS). The goal of the symposium was to highlight how collaboration and teamwork within healthcare can make a difference to improve patient outcomes in thoracic surgery.

Although evidence-based guidelines can provide a framework for an optimized patient recovery pathway, emphasizing the role of a multidisciplinary healthcare team, there are still obstacles to overcome regarding the effective implementation of harmonized perioperative care protocols (1-3).

As data regarding the benefits of enhanced recovery after surgery $\left(\right.$ ERAS $^{\circledR}$ ) pathways on complication rates, lengthof-stay (LOS), and cost continues to accumulate (4-6), some authors underscore the importance of simplifying protocols, focusing on the core pillars of ERAS ${ }^{\circledR}$ to shape the future directions of integrated healthcare in thoracic surgery (7). These core pillars include opioid avoidance, euvolemia, early mobilization, and early feeding, among others. The implementation of an ERAS $^{\circledR}$ strategy can improve patient outcomes and streamline care, but it relies on effective teamwork and active leadership to be successful $(8,9)$.

In this event aimed at thoracic surgeons, several key topics were addressed, including:

* Return to activities of daily living and its importance for patients;

* Patient satisfaction as a quality indicator;

* Early mobilization after surgery;

* Post-surgical pain management;

* The role of chest drainage on ERAS $^{\circledR}$;

* The importance of teamwork to improve patient outcomes.

This report summarizes the discussions, lessons learned, and future directions in patient care and recovery after thoracic surgery.

\section{Presentation 1: the role of physical conditioning before and after surgery for lung cancer}

\section{Speaker: Prof. Vinicius Cavalberi}

The introductory session of the symposium discussed the effectiveness of physical exercise training in lung cancer, and how it is increasingly becoming a part of clinical management in this patient population. Professor Cavalheri started by addressing exercise training before surgery, also called pre-habilitation. He noted that approximately half of the patients with lung cancer also have underlying chronic lung diseases, mainly chronic obstructive pulmonary disease, which further compromise the patients' lung pulmonary mechanics and gas exchange, affecting exercise capacity and consequently increasing the risk for both post-operative pulmonary complications and mortality.

There is a major concern about delaying treatment or surgery for exercise training, since there is no evidence for it, but patients can be enrolled in a physical exercise program while they're waiting for surgery. Usually, this strategy results in one to four weeks of exercise training, mainly aerobic. The aim is to increase $\mathrm{VO}_{2} \max$, and the training frequency can go up to five days a week. According to recent clinical trials and a systematic review (10-12), the use of pre-habilitation exercise was important to preserve exercise capacity, which ultimately resulted in a $67 \%$ reduction on the risk of developing post-operative pulmonary complications in patients that exercised before surgery, compared to patients who did not exercise.

After surgery, the aim of exercise training is slightly different. The focus of post-operative exercise training is to improve the health outcomes affected by surgery, to promote health and develop a positive exercise behavior in patients. Professor Cavalheri noted that while patients understand there is a risk of post-operative pulmonary complications, they value the likelihood of a physical impairment as much more important and undesirable than pulmonary complications (13). This physical impairment is caused by a reduction in $\mathrm{FEV}_{1}$ and $\mathrm{VO}_{2}$ peak after surgery, and this is why early mobilization and physical exercise is important.

The benefits of post-operative exercise training include an increase in $\mathrm{VO}_{2}$ peak, longer distance covered in the six-minute walk distance test, reduction in breathlessness, increased muscle strength, and greater quality of life $(14,15)$. In face of these improvements, the question that naturally comes next is: how soon should patients start exercising after surgery? As soon as possible. The results of a randomized clinical trial involving 235 patients with operable lung cancer showed that patients who initiated post-operative exercise training early had less fatigue by 14 weeks, when compared with those who initiated the training program later (16).

This is an important point, and one where digital chest drainage systems can have a positive impact on patients' lives. Having a portable drainage system allows the patient to be out of bed and walking shortly after surgery, to go 
home early and continue their physical exercise programs. Furthermore, reducing the patients' hospital stay will not only be beneficial for each individual patient, but also for those waiting for surgery, as better discharge planning allows for improved turnaround time in hospital care.

\section{Presentation 2: enhanced recovery in thoracic surgery-the anesthesiology point of view}

\section{Speaker: Prof. Dr. Mert Şentürk}

In his presentation, Prof. Dr. Şentürk underscored the importance of anesthesiology as an important element of ERAS ${ }^{\circledast}$ multidisciplinary approach and explored the difficulties in implementation of ERAS ${ }^{\circledR}$ protocols in thoracic surgery.

For some authors, the usefulness of ERAS ${ }^{\circledR}$ protocols is questionable in certain conditions (17), since this area is perceived as still being in its infancy. This is mainly due to the outcomes chosen in many studies, since there is a focus on visual analogue scales (VAS) to measure the effects of analgesia on pain and on intraoperative $\mathrm{PaO}_{2}$ to assess mechanical ventilation strategies. Another difficulty in the adequate implementation of $\mathrm{ERAS}^{\circledR}$ is the compliance with protocols.

One study (18) showed that even in optimal conditions, overall ERAS ${ }^{\circledR}$ compliance was only $76 \%$. Nonetheless, the authors of this study were able to identify four elements that are essential to reduce the rate of post-operative complications: early removal of chest tubes, use of electronic drainage, opioid cessation on day 3 , and early feeding. It is worth noting that more complex protocols tend to cause more difficulties with compliance, so we should strive for simpler ERAS ${ }^{\circledR}$ protocols in thoracic surgery to improve adherence.

One crucial component to improve patients' outcomes is pain management. Prof. Dr. Şentürk noted that the target should not be to achieve a completely painless patient, and the measured outcome should be more than patient comfort. Pain is a multi-component issue that benefits from a multimodal analgesia strategy, he noted. As an example, Prof. Dr. Şentürk mentioned that, in his opinion, chest drain pain deserves more attention. It is already known that using a single chest tube results in less pain compared to double chest tube (2), and that digital drainage systems lead to a shorter duration of chest drain placement, which can probably shorten the painful period too.

Another topic discussed was the possible impact of pre-habilitation exercise programs on compliance with protocols. Although a longer period of exercise before surgery can be seen as beneficial, there are human factors to consider, and there should be a balance between and effective length of the program, and the need to keep the patient engaged. In the post-surgery rehabilitation exercise program, the focus goes to an early mobilization of the patient. Here, the burden of chest drainage can be reduced by using portable, digital chest drainage systems to promote patient's mobility.

In conclusion, to move forward with ERAS ${ }^{\circledR}$ protocols in thoracic surgery, there's a need for a higher number of adequately powered clinical studies, with pragmatic, rational outcomes, and involving a multidisciplinary healthcare team. In addition, simpler protocols will result in better compliance.

\section{Presentation 3: pain management after VATS}

\section{Speaker: Shanda H. Blackmon, MD, MPH, FACS}

This session, presented by Dr. Shanda H. Blackmon, converged several topics regarding pain management after thoracic surgery, including strategies to reduce opioid-related adverse events, the use of regional blocks to minimize opioid use, and the role of $\mathrm{ERAS}^{\circledR}$ in pain management.

Pain pathways are complex, with multiple mechanisms involved, including nociceptive and neuropathic ones. There are several factors that can impact pain following a thoracic surgery, such as muscle splitting, injury to the parietal pleura, and the presence of the chest tube (19). Furthermore, surgery releases inflammatory mediators, such as prostaglandins and histamine, which enhance the activity of nociceptors. Given the multifactorial origin of pain, the logical step in tackling post-thoracic lung resection pain is to target different pathways, Dr. Blackmon said.

The pharmacological approaches to pain modulation include the use of gabapentin, acetaminophen (paracetamol), nonsteroidal anti-inflammatory drugs, ketamine, opioids, and local anesthetics. Despite the existence of alternatives, opioids are still widely used after major surgery. Patients who undergo thoracotomy have a 2.5 -fold increase in prolonged opioid use risk, with those undergoing videoassisted thoracoscopic surgery (VATS) not far behind, with a 1.95 -fold risk increase $(20,21)$. In addition to opioid-related adverse events such as respiratory depression, somnolence, and nausea or vomiting experienced by patients, there are 
also public health effects to be considered. The risk of addiction is higher than what clinicians suspected, there are still unknown effects on survival in lung cancer patients, and there is growing concern about the quantity of unused opioids that remain in the community (22).

One alternative to opioids is the use of regional blocks with local analgesia. Dr. Blackmon explained several techniques, such as the thoracic paravertebral block (TPVB), the serratus anterior plane block (SAPB), and the intercostal nerve block (ICNB), noting that the paravertebral and intercostal seem to have the least side effects combined with the best pain control profile (23).

Regarding ERAS ${ }^{\circledR}$, Dr. Blackmon referred that these programs are associated with many short-term outcome benefits, such as reduction in post-operative morbidity, reduction in pain, reduction in length of stay, and overall patient satisfaction $(24,25)$. Regarding pain in particular, the shorter the duration of the air leak and the chest tube duration, the less pain is experienced by patients. As part of the pain control, the aim is to seal the lung and remove the chest tube as soon as possible, and use the smallest chest tube possible and minimal suction. ERAS ${ }^{\circledR}$ recommendations to minimize pain include the use of digital drainage systems, which only use suction as needed (as opposed to traditional wall suction systems which use constant suction), using a single chest tube (26), and early patient mobilization (1).

Digital drainage systems are sophisticated devices delivering a regulated form of negative pressure by adjusting the level of suction to the pre-set negative pressure. In case the pre-set intrapleural negative pressure is reached the device stop working and act as a monitoring device recording both the effusion and air leak. One of the main advantages of these devices is the fact they record objective information about air leak showing trends along with instantaneous measurements which improve and standardize clinical decision making. There is growing evidence regarding the benefits of digital drainage systems, which include balanced suction, easy to read displays, promotion of mobilization and communication, 24-hour registration of fluid and air, and the possibility of early removal of the chest tube, resulting in less pain for the patient. The benefits of lower suction have been demonstrated in a study by Holbek et al. (27), where the results show that drainage duration, time to air leak cessation, and time to drain removal were all significantly reduced by utilization of a lower suction on the chest tube, accomplished by the use of a digital drainage system (Thopaz+, Medela AG, Switzerland). One study explored the possibility of omitting the chest tube altogether, and there is some indication that it may lead to shorter duration of stay and less pain, if applied in carefully selected patients (i.e., lung biopsy, uncomplicated wedge for peripheral nodules, excision of mediastinal tumours, in patients at low risk for air leak) (28). However, there is a higher risk of drain re-insertion or thoracocentesis in patients who do not have chest drains.

Dr. Blackmon ended her presentation by saying that there is a need to reduce pain though the use of more minimally invasive surgery, lower suction, digital drainage, and a combined approach to pain management, including regional blockade and ERAS $^{\circledR}$ protocols.

\section{Presentation 4: the lung cancer surgical journey-patient perspectives and values}

\section{Speaker: Cecilia Pompili, MD PbD}

On the final presentation of the symposium, Dr. Pompili shared her knowledge about incorporating patient-reported outcomes and experiences in clinical practice, including the difference between outcomes and experiences, the challenges in the implementation of these measures, and the future directions of patient-reported outcomes.

The success of patient-centered care can be assessed by exploring the patient experiences, which capture the patient's view of what happened while they received their care. The patient experiences are, therefore, an indirect indicator of the quality of patient care, and this is why the investigation of patient-reported experience measures (PREMs) are an emerging area of research. Patient-reported outcome measures (PROMs) are used to capture the impact of an illness or health condition directly from the patient, without interpretation by a clinician or caregiver. When used together, PREMs and PROMs can support patient-centered care and inform about the quality of care and services.

Another indicator that can help to understand the patient's perspectives is the health-related quality of life (HRQoL), a subjective and multidimensional concept that assesses the functional effect of a medical condition and/ or its treatment on the patient's quality of life. According to recent research, the regular compilation of PROMs and HRQoL can help detect, manage and monitor problems, while also facilitating patient-doctor communication $(29,30)$. However, an essential requirement for their correct implementation is training, for both patients and clinicians (31), as well as the use of a standardized and 
validated tool.

Regarding thoracic surgery, despite the recognition that PROMs provide essential data in perioperative care which could help drive the treatment course for each individual patient, influence continuous improvement in health system, and impact patient's long-term satisfaction and wellbeing, a recent ESTS survey (32) revealed that more than half the respondents were not collecting any quality of life data in daily practice. It is worth to note that while it is crucial to identify predictors of potential decline of quality of life after surgery, is has been demonstrated that for patients the major concern may not be perioperative morbidity and mortality, but rather to not be able to resume an acceptable daily lifestyle. So, again, the use of both PROMs and PREMs (and their impact on HRQoL measures) can have a positive influence in the pre-surgical decision-making process. Namely, it has been shown that pre-operative quality of life can help predict survival outcomes following pulmonary resection (33).

An important aspect of patient experience of care is patient satisfaction, Dr. Pompili says. Patient satisfaction can be used to compare different technologies, with several studies pointing to an association between satisfaction and outcomes after a surgery. For example, a recent study assessing the impact of digital versus traditional drainage devices on patient satisfaction showed that patients managed with digital drainage systems had higher satisfaction scores compared with those managed with traditional devices, in particular regarding comfort, portability, and convenience of use (34).

As for the future of PROMs in thoracic surgery, Dr. Pompili observed that the implementation of electronic means of capturing and reporting them will empower patients and give surgeons the opportunity to use these measures in real-life, effectively placing the patient at the center of care.

\section{Conclusions}

The contributions made at the symposium support the view that teamwork and ERAS $^{\circledR}$ protocols are essential pillars of patient-centered care in thoracic surgery. Among the key learnings that emerged from the presentations are the importance of taking the patient's experiences into account, including what they value the most after surgery, and how technology can enable better care, especially regarding chest drainage systems. There were two main ideas that were considered pivotal to achieve optimal care: (I) the use of simple, easily implementable perioperative protocols and practices to improve compliance from both patients and the healthcare team, and (II) promote a better recovery with early mobilization and reducing the patient's levels of pain.

In conclusion, the presentations in this symposium allowed for a better understanding of the many components involved in patient-centered care in thoracic surgery, along with the future directions for ERAS ${ }^{\circledR}$. Among the several topics addressed, the relevance of chest drainage and its impact on patient recovery was highlighted as one that can affect several indicators, from outcomes to satisfaction.

\section{Acknowledgments}

Medical writing assistance was provided by Diana Ribeiro, MPharm, and funded by Medela AG, Switzerland.

Funding: This symposium was supported by Medela AG, Switzerland.

\section{Footnote}

Peer Review File: Available at https://jtd.amegroups.com/ article/view/10.21037/jtd-21-1763/prf

Conflicts of Interest: All authors have completed the ICMJE uniform disclosure form (available at https://jtd.amegroups. com/article/view/10.21037/jtd-21-1763/coif). AB serves as an unpaid Associate Editor-in-Chief of Journal of Thoracic Disease. CP serves as an unpaid editorial board member of Fournal of Thoracic Disease from September 2016 to August 2022. AB reports receiving consulting fees and honoraria for lectures and work with advisory boards from Astra Zeneca, Becton Dickinson-BD, Ethicon, Medtronic. In addition, he received Honorarium from Medela to organize and chair the symposium Patient-centered care in thoracic care: a holistic approach that was held on June 22, 2021, as a virtual event. SHB and VC reports receiving an Honorarium from Medela to present at the symposium Patient-centered care in thoracic care: a holistic approach that was held on June 22, 2021, as a virtual event. CP reports receiving an Honorarium from Medela to present at the symposium Patient-centered care in thoracic care: a holistic approach that was held on June 22, 2021, as a virtual event. She also received a speaker fee from BD for a lecture at an educational event. The other author has no conflicts of interest to declare.

Ethical Statement: The authors are accountable for all 
aspects of the work in ensuring that questions related to the accuracy or integrity of any part of the work are appropriately investigated and resolved.

Open Access Statement: This is an Open Access article distributed in accordance with the Creative Commons Attribution-NonCommercial-NoDerivs 4.0 International License (CC BY-NC-ND 4.0), which permits the noncommercial replication and distribution of the article with the strict proviso that no changes or edits are made and the original work is properly cited (including links to both the formal publication through the relevant DOI and the license). See: https://creativecommons.org/licenses/by-nc-nd/4.0/.

\section{References}

1. Batchelor TJP, Rasburn NJ, Abdelnour-Berchtold E, et al. Guidelines for enhanced recovery after lung surgery: recommendations of the Enhanced Recovery After Surgery (ERAS®) Society and the European Society of Thoracic Surgeons (ESTS). Eur J Cardiothorac Surg 2019;55:91-115.

2. Piccioni F, Droghetti A, Bertani A, et al. Recommendations from the Italian intersociety consensus on Perioperative Anesthesia Care in Thoracic surgery (PACTS) part 1: preadmission and preoperative care. Perioper Med (Lond) 2020;9:37.

3. Ljungqvist $\mathrm{O}$, de Boer HD, Balfour A, et al. Opportunities and Challenges for the Next Phase of Enhanced Recovery After Surgery: A Review. JAMA Surg 2021;156:775-84.

4. Li S, Zhou K, Che G, et al. Enhanced recovery programs in lung cancer surgery: systematic review and metaanalysis of randomized controlled trials. Cancer Manag Res 2017;9:657-70.

5. Gort N, van Gaal BGI, Tielemans HJP, et al. Positive effects of the enhanced recovery after surgery (ERAS) protocol in DIEP flap breast reconstruction. Breast 2021;60:53-7.

6. Wang C, Lai Y, Li P, et al. Influence of enhanced recovery after surgery (ERAS) on patients receiving lung resection: a retrospective study of 1749 cases. BMC Surg 2021;21:115.

7. Merchea A, Larson DW. Enhanced Recovery After Surgery and Future Directions. Surg Clin North Am 2018;98:1287-92.

8. Byrne BE, Faiz OD, Bottle A, et al. A Protocol is not Enough: Enhanced Recovery Program-Based Care and Clinician Adherence Associated with Shorter Stay After Colorectal Surgery. World J Surg 2021;45:347-55.
9. Zorrilla-Vaca A, Stone AB, Ripolles-Melchor J, et al. Institutional factors associated with adherence to enhanced recovery protocols for colorectal surgery: Secondary analysis of a multicenter study. J Clin Anesth 2021;74:110378.

10. Stefanelli F, Meoli I, Cobuccio R, et al. High-intensity training and cardiopulmonary exercise testing in patients with chronic obstructive pulmonary disease and nonsmall-cell lung cancer undergoing lobectomy. Eur J Cardiothorac Surg 2013;44:e260-5.

11. Licker M, Karenovics W, Diaper J, et al. Short-Term Preoperative High-Intensity Interval Training in Patients Awaiting Lung Cancer Surgery: A Randomized Controlled Trial. J Thorac Oncol 2017;12:323-33.

12. Cavalheri V, Granger C. Preoperative exercise training for patients with non-small cell lung cancer. Cochrane Database Syst Rev 2017;6:CD012020.

13. Cykert S, Kissling G, Hansen CJ. Patient preferences regarding possible outcomes of lung resection: what outcomes should preoperative evaluations target? Chest 2000;117:1551-9.

14. Granger CL, Holland AE, Gordon IR, et al. Minimal important difference of the 6-minute walk distance in lung cancer. Chron Respir Dis 2015;12:146-54.

15. Cavalheri V, Burtin C, Formico VR, et al. Exercise training undertaken by people within 12 months of lung resection for non-small cell lung cancer. Cochrane Database Syst Rev 2019;6:CD009955.

16. Quist M, Sommer MS, Vibe-Petersen J, et al. Early initiated postoperative rehabilitation reduces fatigue in patients with operable lung cancer: A randomized trial. Lung Cancer 2018;126:125-32.

17. Brunelli A, Thomas C, Dinesh P, et al. Enhanced recovery pathway versus standard care in patients undergoing videoassisted thoracoscopic lobectomy. J Thorac Cardiovasc Surg 2017;154:2084-90.

18. Forster C, Doucet V, Perentes JY, et al. Impact of Compliance With Components of an ERAS Pathway on the Outcomes of Anatomic VATS Pulmonary Resections. J Cardiothorac Vasc Anesth 2020;34:1858-66.

19. Barbeito A, Shaw AD, Grichnik K. Thoracic anesthesia. New York, NY: McGraw-Hill Professional, 2012.

20. Clarke H, Soneji N, Ko DT, et al. Rates and risk factors for prolonged opioid use after major surgery: population based cohort study. BMJ 2014;348:g1251.

21. Brummett CM, Waljee JF, Goesling J, et al. New Persistent Opioid Use After Minor and Major Surgical Procedures in US Adults. JAMA Surg 2017;152:e170504. 
22. Wheeler M, Oderda GM, Ashburn MA, et al. Adverse events associated with postoperative opioid analgesia: a systematic review. J Pain 2002;3:159-80.

23. Kaplowitz J, Papadakos PJ. Acute pain management for video-assisted thoracoscopic surgery: an update. J Cardiothorac Vasc Anesth 2012;26:312-21.

24. Mena GE, Zorrilla-Vaca A, Vaporciyan A, et al. Intraoperative Dexmedetomidine and Ketamine Infusions in an Enhanced Recovery After Thoracic Surgery Program: A Propensity Score Matched Analysis. J Cardiothorac Vasc Anesth 2021. [Epub ahead of print].

25. Zorrilla-Vaca A, Rice D, Brown JK, et al. Sustained reduction of discharge opioid prescriptions in an enhanced recovery after thoracic surgery program: A multilevel generalized linear model. Surgery 2022;171:504-10.

26. Zorrilla-Vaca A, Feldman H, Antonoff M, et al. Single Chest Drain Practice Reduces Discharge Opioid Prescriptions in Thoracic Surgery. Thorac Cardiovasc Surg 2021. [Epub ahead of print].

27. Holbek BL, Christensen M, Hansen HJ, et al. The effects of low suction on digital drainage devices after lobectomy using video-assisted thoracoscopic surgery: A randomized controlled trial. Eur J Cardiothorac Surg 2019;55:673-81.

28. Huang L, Kehlet H, Holbek BL, et al. Efficacy and safety of omitting chest drains after video-assisted thoracoscopic surgery: a systematic review and meta-analysis. J Thorac Dis 2021;13:1130-42.

Cite this article as: Brunelli A, Blackmon SH, Sentürk M, Cavalheri V, Pompili C. Patient-centred care in thoracic surgery: a bolistic approach —A review of the subjects of enhanced recovery after surgery, rehabilitation, pain management and patientreported outcome measures in thoracic surgery. J Thorac Dis 2022;14(2):546-552. doi: 10.21037/jtd-21-1763
29. Greenhalgh T, Hinton L, Finlay T, et al. Frameworks for supporting patient and public involvement in research: Systematic review and co-design pilot. Health Expect 2019;22:785-801.

30. Graupner C, Kimman ML, Mul S, et al. Patient outcomes, patient experiences and process indicators associated with the routine use of patient-reported outcome measures (PROMs) in cancer care: a systematic review. Support Care Cancer 2021;29:573-93.

31. Giesinger JM, Efficace F, Aaronson N, et al. Past and Current Practice of Patient-Reported Outcome Measurement in Randomized Cancer Clinical Trials: A Systematic Review. Value Health 2021;24:585-91.

32. Pompili C, Novoa N, Balduyck B, et al. Clinical evaluation of quality of life: a survey among members of European Society of Thoracic Surgeons (ESTS). Interact Cardiovasc Thorac Surg 2015;21:415-9.

33. Pompili C, Salati M, Refai M, et al. Preoperative quality of life predicts survival following pulmonary resection in stage I non-small-cell lung cancer. Eur J Cardiothorac Surg 2013;43:905-10.

34. Pompili C, Detterbeck F, Papagiannopoulos K, et al. Multicenter international randomized comparison of objective and subjective outcomes between electronic and traditional chest drainage systems. Ann Thorac Surg 2014;98:490-6; discussion 496-7. 